Check for updates

Cite this: RSC Adv., 2017, 7, 26120

Received 10th March 2017

Accepted 1st May 2017

DOI: 10.1039/c7ra02910a

rsc.li/rsc-advances

\section{Ternary NiCoP nanoparticles assembled on graphene for high-performance lithium-ion batteries and supercapacitors $\uparrow$}

\author{
Chunde Wang, iD abcd Yinyin Qian, ${ }^{\text {abcd }}$ Jing Yang, ${ }^{\text {abcd }}$ Shiqi Xing, ${ }^{\text {abcd }}$ Xu Ding ${ }^{\text {abcd }}$ \\ and Qing Yang*abcd
}

\begin{abstract}
Transition metal phosphides have received considerable interest for electrochemical energy storage/ conversion and catalysis. In this work, we designed a unique hybrid of NiCoP nanoparticles adhered on quasi-planar structured graphene by assembling $8.5 \mathrm{~nm}$ ternary NiCoP nanoparticles on graphene through a solution-phase self-assembly strategy. The NiCoP catalyst in the form of small-size particles wrapped in graphene provided more active sites, a buffer for volume alteration and enhanced conductivity for electrochemical reactions. Typically, the hybrid catalyst demonstrates a high specific capacity of around $532 \mathrm{~mA} \mathrm{~h} \mathrm{~g}^{-1}$, excellent cycling stability and superior rate performance when the hybrid material is evaluated as an anode material for lithium-ion batteries, and it shows excellent electrochemical properties with a specific capacitance of $646 \mathrm{~F} \mathrm{~g}^{-1}$ at $4 \mathrm{~A} \mathrm{~g}^{-1}$, maintaining $91 \%$ of this initial value after 2000 cycles functioning as a supercapacitor.
\end{abstract}

\section{Introduction}

With the increasing depletion of non-renewable resources and the growing environmental pollution due to the use of fossil fuels, the global energy crisis is worsening. Developing new energy sources with high power density at low cost has attracted tremendous interest. ${ }^{\mathbf{1}, 2}$ Lithium ion batteries (LIBs) and supercapacitors are promising energy conversion/storage and power output devices for meeting the demands of digital communications, portable electronic devices and hybrid vehicles. ${ }^{3-7}$ Recently, much effort has been devoted to high-capacity materials with superior long-term stability to meet the growing demands for flexible and practical electric products. ${ }^{8-12}$ To the best of our knowledge, the performance of LIBs and supercapacitors is largely dependent on the structure and morphology of the electrode materials. ${ }^{13-15}$ As a consequence, it is of great significance to seek appropriate nanostructured materials. In the past three decades, a series of nanomaterials with different dimensionalities, such as 0D quantum dots, ${ }^{16,17}$

${ }^{a}$ Hefei National Laboratory of Physical Sciences at the Microscale, University of Science and Technology of China (USTC), Hefei 230026, Anhui, P. R. China.E-mail: qyoung@ ustc.edu.cn; Fax: +86-551-63606266; Tel: +86-551-63600243

${ }^{b}$ Department of Chemistry, USTC, Hefei 230026, Anhui, P. R. China

${ }^{c}$ Laboratory of Nanomaterials for Energy Conversion, USTC, Hefei 230026, Anhui, P. R. China

${ }^{d}$ Department of Physics, USTC, Hefei 230026, Anhui, P. R. China

$\dagger$ Electronic supplementary information (ESI) available: Materials including methods, TEM images of the NiCoP/G hybrid after the treatment, XRD and EDX of the samples, Raman spectrum of the NiCoP/G hybrid, discharge-charge voltage profiles of the NiCoP/G hybrid. See DOI: 10.1039/c7ra02910a
1D nanowires, ${ }^{\mathbf{1 8 , 1 9}}$ 2D nanosheets ${ }^{\mathbf{2 0 - 2 2}}$ and 3D network-like structures, ${ }^{23-26}$ have been found and applied in electrode materials.

Transition metal phosphides (TMPs), especially binary TMPs $\left(\mathrm{MP}_{x}\right.$, where $\mathrm{M}=\mathrm{Cu}, \mathrm{Fe}, \mathrm{Co}$, etc. $)$, have been considered as alternative anode materials for LIBs because of their exceptionally high gravimetric storage capacities (500-1800 $\mathrm{mA} \mathrm{h} \mathrm{g}^{-1}$ ) compared to conventional carbon-based anode materials, their low lithiation-delithiation potentials and the low cost of the raw materials. ${ }^{27-33}$ However, the practical application of LIBs is often hindered on account of various critical challenges. First of all, the main challenge is the large volume alteration of TMPs during charge/discharge: the lithium ion-coupled charge transfer reactions in TMPs induce large irreversible volume changes, which can cause pulverization of the initial crystal structure and the aggregation of the active material. This process of large volume alteration further leads to poor capacity retention. The second key problem is related to the insulating nature of TMPs, including their binary compounds and multielemental derivatives: the intrinsically low electronic conductivity of such materials tends to limit their practical applications in most cases. Meanwhile, the same problems are encountered in the development of supercapacitors, in addition to some other energy-conversion systems, when TMPs are employed as active electrode materials. To date, to overcome the above limitations of electrode materials with low conductivity and large volume changes, several effective methods have been developed, such as introducing conductive agents (including carbon shells, carbon networks, graphene nanosheets, etc.) and constructing various nanostructures, and it is found that these 
strategies can effectively improve the cycling performance, and especially the rate capability, of the electrode materials. ${ }^{\mathbf{3 4 - 3 8}}$

As previously reported, graphene is a two-dimensional single-layer sheet of carbon atoms, and is particularly attractive as a substrate material to host nano-materials based on metal compounds due to its mechanical strength, large surface area and high electronic conductivity. ${ }^{39-41}$ In the present work, we demonstrate that monodisperse ternary NiCoP nanoparticles can be self-assembled on graphene (termed NiCoP/G hybrid) at room temperature by a solution-phase method, and our electrode materials exhibit a high capacity and excellent cycling stability for LIBs and supercapacitors. As a proof of concept, the introduction of carbon materials into the nanocrystals gives rise to a high electrochemical performance compared with that of the pure phase crystals. We present electrochemical data demonstrating the good performance of the NiCoP/G hybrid electrode, which delivered a discharge capacity of $532 \mathrm{~mA} \mathrm{~h} \mathrm{~g}^{-1}$ during the 100th cycle at a charge/ discharge rate of $100 \mathrm{~mA} \mathrm{~g}^{-1}$, and good rate performance. Meanwhile, the NiCoP/G hybrid showed excellent electrochemical properties with a specific capacitance of $646 \mathrm{~F} \mathrm{~g}^{-1}$ at 4 $\mathrm{A} \mathrm{g}^{-1}$ and maintained $91 \%$ of this initial value after 2000 cycles.

\section{Results and discussion}

Monodisperse NiCoP nanoparticles (NPs) were grown via a reflux process with some modification in high quality and high yield, and they were assembled on a graphene surface through a solution-phase self-assembly method (see the ESI $\uparrow$ for

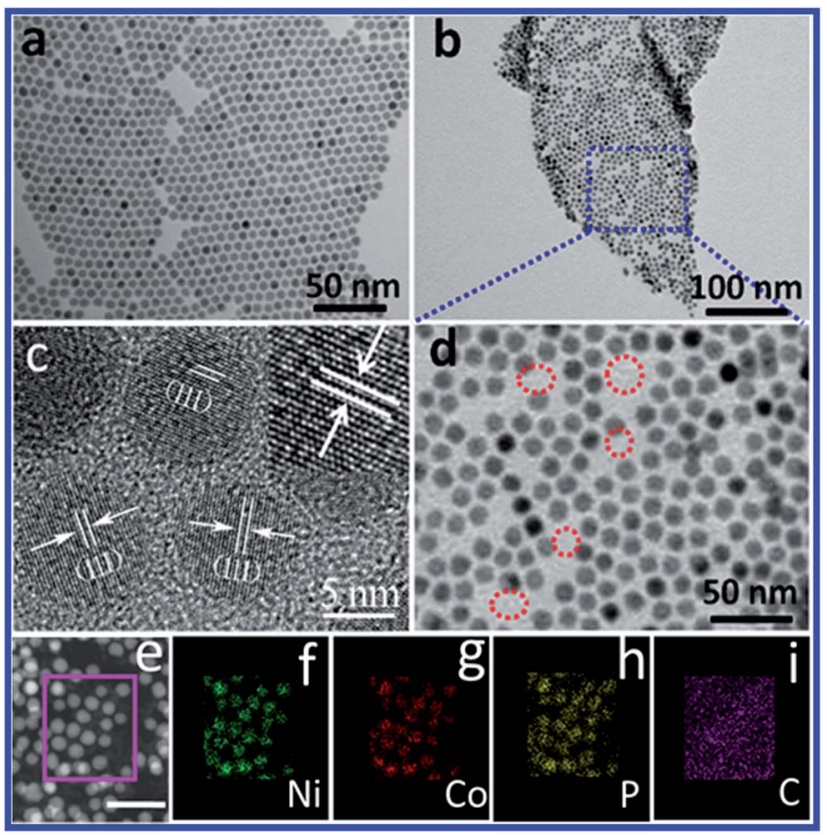

Fig. 1 (a) TEM image of the NiCoP NPs assembled on an amorphous carbon surface, (b) low-magnified, (d) high-magnified TEM images of the NiCoP/G hybrid, (c) HRTEM image of the NiCoP NPs. (e) HAADFSTEM image of the NiCoP/G hybrid, scale bars represent $20 \mathrm{~nm}$. (f-i) Elemental maps of Ni (green), Co (red), P (yellow) and C (purple), respectively. details). Both NiCoP NPs and NiCoP/G hybrid were characterized by transmission electron microscopy (TEM). Fig. 1a is a typical TEM image of the NiCoP NPs deposited on an amorphous carbon-coated copper grid, and they show a narrow size distribution with a diameter of $8.5 \pm 0.5 \mathrm{~nm}$. An SEM image of the NiCoP/G hybrid is provided in Fig. S1. $\dagger$ It is clear to observe the well-dispersed NiCoP NPs deposited uniformly on the surface of the graphene throughout the whole morphology. Fig. $1 \mathrm{~b}$ and $\mathrm{d}$ show TEM images of the NiCoP/G hybrid at different magnifications, confirming that no NPs were detached from the graphene substrate. In the hybrid material, the NiCoP NPs selectively and densely grew on the graphene sheets (the exposed graphene is marked by dashed red circles). During synthesis, to ensure the particles adhesion on graphene, the samples of NPs were washed with acetic acid at $70{ }^{\circ} \mathrm{C}$ overnight and annealed at $100{ }^{\circ} \mathrm{C}$ under a gas mixture of $\mathrm{Ar}+5 \% \mathrm{H}_{2}$ for $1 \mathrm{~h}$ to remove the surfactant around each NiCoP NP. The NiCoP NPs in the NiCoP/G hybrid did not show any morphological changes after the treatment (Fig. S2 $\dagger$ ). As seen in Fig. 1c, high-resolution TEM (HRTEM) images reveal interplanar spacings of $0.22 \mathrm{~nm}$, which can be assigned to the (111) plane of the hexagonal NiCoP crystal structure, consistent with the X-ray diffraction (XRD) data of NiCoP (JCPDS 77-1716, Fig. S3†). The inset in Fig. 1c is the magnified lattice fringe of the NiCoP NPs. To further demonstrate the element distribution, high-angle annular darkfield scanning TEM energy dispersive X-ray spectroscopic (HAADF-STEM-EDX) elemental mappings are provided in Fig. 1e-i, verifying the co-existence of $\mathrm{Ni}, \mathrm{Co}, \mathrm{P}$ and $\mathrm{C}$ in the $\mathrm{NiCoP} / \mathrm{G}$ hybrid. In addition, the elemental ratio of $\mathrm{Ni}: \mathrm{Co}: \mathrm{P}$ in the product was determined to be about $1: 1: 1$, corresponding well with its stoichiometric ratio (Fig S4 $\dagger$ ).

The chemical compositions and states of the NiCoP/G hybrid were investigated by X-ray photoelectron spectroscopy (XPS). The corresponding XPS survey scan shows that the sample contains the elements $\mathrm{Ni}, \mathrm{Co}, \mathrm{P}$ and $\mathrm{C}$. The high resolution XPS spectra of the elements Ni, Co, P and C from the NiCoP/G hybrid are shown in Fig. 2a-d. In the Ni 2p (Fig. 2a) and Co 2p (Fig. 2b)

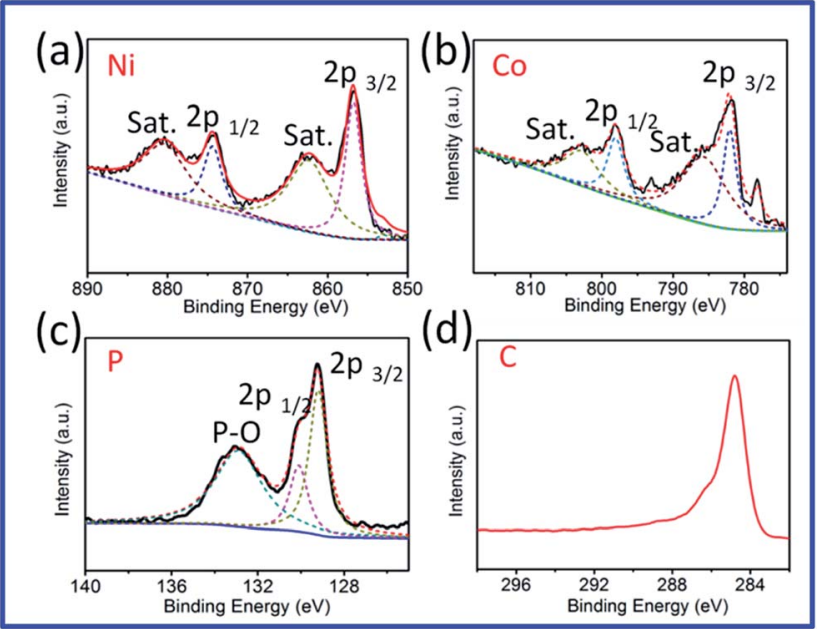

Fig. 2 XPS spectra of (a) Ni 2p, (b) Co 2p, (c) P 2p and (d) C regions for the NiCoP/G hybrid. 
spectra, two pairs of spin-orbit doublets and two shake-up (satellites, shortened as sat.) excitations were obtained, indicating the existence of $\mathrm{Ni}^{2+} / \mathrm{Ni}^{3+}$ and $\mathrm{Co}^{2+} / \mathrm{Co}^{3+}$ in the samples, respectively. ${ }^{\mathbf{4 2 4 3}}$ The peaks at 853.2 and $778.4 \mathrm{eV}$ are close to those of zero valence $\mathrm{Ni}(852.8 \mathrm{eV})$ and $\mathrm{Co}(778.1 \mathrm{eV}){ }^{43,44}$ It is therefore suggested that the $\mathrm{Ni}$ and Co species in NiCoP have a very small positive charge. Accordingly, the high-resolution spectrum of the $\mathrm{P} 2 \mathrm{p}$ region shows two peaks at 130.4 and $129.3 \mathrm{eV}$, reflecting the binding energy of $\mathrm{P} 2 \mathrm{p}_{1 / 2}$ and $\mathrm{P} 2 \mathrm{p}_{3 / 2}$ (Fig. 2c). ${ }^{45,46}$ The peak at $129.3 \mathrm{eV}$ is at lower energy than that of elemental $\mathrm{P}(130.2 \mathrm{eV})$, so the $\mathrm{P}$ species in NiCoP has a very small negative charge. In addition, the peaks at $133.5 \mathrm{eV}$ show that the P species (like the $\mathrm{Ni}$ and $\mathrm{Co}$ ) is oxidized due to the exposure of the sample to acid solution and air. As shown in Fig. 2d, the peak at $284.8 \mathrm{eV}$ is ascribed to $\mathrm{C} 1 \mathrm{~s}$. Meanwhile, the Raman spectra of the hybrid (Fig. S5 $\dagger$ ) show two intense peaks located at 1358 and $1588 \mathrm{~cm}^{-1}$, assigned to the D- and G-bands of graphite, respectively, further evidencing the successful modification of graphene in the samples.

Recently, as a new alternative anode material, TMPs have attracted much attention due to their high theoretical capacity. In order to evaluate the $\mathrm{NiCoP} / \mathrm{G}$ hybrid as a potential anode material for lithium ion batteries, CR2016 type coin-cells with lithium foil as a counter electrode were prepared. Fig. S6† shows the first and 100th galvanostatic charge-discharge curves of the $\mathrm{NiCoP} / \mathrm{G}$ hybrid from 0.005 to $3.0 \mathrm{~V}$ at a current density of 100 $\mathrm{mA} \mathrm{g}^{-1}$. The initial discharge and charge specific capacities reached 1034 and $686 \mathrm{~mA} \mathrm{~h} \mathrm{~g}^{-1}$, respectively, indicating irreversible losses of about $33.6 \%$, which may be ascribed to the irreversible formation of a solid electrolyte interlayer (SEI) and the partial destruction of the internal structure. The cyclic voltammogram (CV) profiles of the NiCoP/G hybrid for the first three cycles at a scan rate of $0.1 \mathrm{mV} \mathrm{s}^{-1}$ are shown in Fig. 3a. The

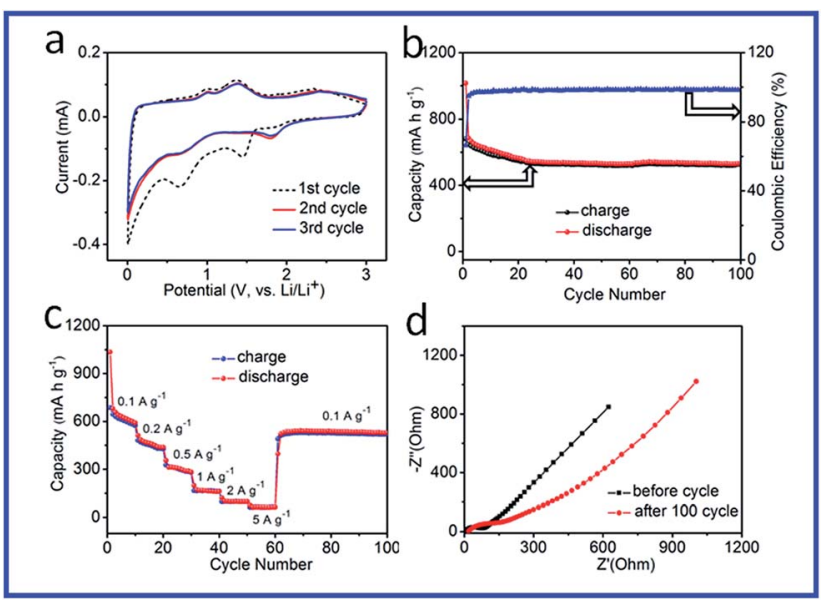

Fig. 3 (a) Cyclic voltammograms of the first three cycles of typical $\mathrm{NiCoP} / \mathrm{G}$ hybrid obtained between 0.005 and $3.0 \mathrm{~V}$ at a scan rate of $0.1 \mathrm{mV} \mathrm{s}^{-1}$. (b) Cycling performance of NiCoP/G hybrid between 0.005 and $3 \mathrm{~V}$ (vs. $\mathrm{Li}^{+} / \mathrm{Li}$ ) at $100 \mathrm{~mA} \mathrm{~g}^{-1}$; (c) rate capability of the NiCoP/G hybrid electrode at various current densities (from $0.1 \mathrm{~A} \mathrm{~g}^{-1}$ to $5 \mathrm{~A} \mathrm{~g}^{-1}$ ). (d) Electrochemical impedance spectra of the NiCoP/G hybrid electrode before cycling and after 100 cycles at $100 \mathrm{~mA} \mathrm{~g}^{-1}$. first discharge for the NiCoP/G hybrid shows a peak at $1.45 \mathrm{~V}$, corresponding to the $\mathrm{Li}^{+}$insertion process. ${ }^{29}$ Another reduction peak located at $0.65 \mathrm{~V}$ corresponds to the decomposition of $\mathrm{NiCoP}$ into metallic $\mathrm{Ni}$ and $\mathrm{Co}$ and the formation of amorphous $\mathrm{Li}_{3} \mathrm{P}$ and an SEI film. During the charging process, the anodic peaks at 1.01 and $1.42 \mathrm{~V}$ are related to the decomposition of the SEI film and $\mathrm{Li}_{3} \mathrm{P}$, and the one at $2.42 \mathrm{~V}$ is attributed to the partially reformed crystalline $\mathrm{Ni}_{2} \mathrm{P}$ phase. ${ }^{47}$ After the initial cycle, the cathodic peaks shift to near 1.80 and $0.71 \mathrm{~V}$, indicating the effect of the SEI film on the $\mathrm{Li}^{+}$kinetic process. A good overlapping of the CV curves from the first and second cycles indicates the reversible Li-storage behavior and high coulombic efficiency.

Stable cyclic performance of the electrode is important for practical application of LIBs. Fig. 3b shows the cyclic performances of the NiCoP/G hybrid electrode at a charge-discharge rate of $100 \mathrm{~mA} \mathrm{~g}^{-1}$. It can be seen that the reversible capacity of the NiCoP/G hybrid slightly decreases in the first cycle and then keeps a stable value and reaches $532 \mathrm{~mA} \mathrm{~h} \mathrm{~g}^{-1}$ after 100 cycles, corresponding to $78.2 \%$ capacity retention compared with the second cycle, and its coulombic efficiency (the blue dots in Fig. 3 b) remains consistently at $\sim 97 \%$. As is known, the major drawback that limits the practical application of TMPs is the drastic capacity fading during the cycle process. ${ }^{31}$ Here, the specific capacity retention has been greatly improved for the NiCoP NPs by incorporating graphene sheets. The rate performance of the electrode is also important for the application of LIBs. The tested rate performance (Fig. 3c) shows that the $\mathrm{NiCoP} / \mathrm{G}$ hybrid electrode exhibits excellent cycling stability. In detail, the reversible capacity of the NiCoP/G hybrid decreases from 680 to $512,354,170,120$, and $74 \mathrm{~mA} \mathrm{~h} \mathrm{~g}{ }^{-1}$ when the current density increases from 0.1 to $0.2,0.5,1,2$, and $5 \mathrm{~A} \mathrm{~g}^{-1}$, respectively. After the current density decreases back to $100 \mathrm{~mA}$ $\mathrm{g}^{-1}$, the discharge capacity of the $\mathrm{NiCoP} / \mathrm{G}$ electrode recovers to $528 \mathrm{~mA} \mathrm{~h} \mathrm{~g}^{-1}$. In order to further understand the reason for this outstanding performance of the NiCoP/G hybrid electrode, an AC impedance measurement was conducted. The electrochemical impedance spectra (EIS) of the as-prepared hybrid electrode tested before cycling and after 100 cycles at $0.1 \mathrm{~A} \mathrm{~g}^{-1}$ are presented in Nyquist plots (Fig. 3d). The plots consist of a semicircle in the high frequency region and a sloped line in the low frequency region, which can be attributed to charge transfer processes and the diffusion of lithium ions, respectively. It can be seen that the $\mathrm{NiCoP} / \mathrm{G}$ hybrid exhibits good electron-transport during the cycling process due to the introduction of graphene. For comparison, the cycling performance of the NiCoP NPs was tested by investigating the charge/ discharge process under the same conditions (as seen in Fig. S7a †).

To further evaluate the capacitance performance of the $\mathrm{NiCoP} / \mathrm{G}$ hybrid electrode material, $\mathrm{CV}$ and galvanostatic charge-discharge tests of the NiCoP/G hybrid in a $2.0 \mathrm{M} \mathrm{KOH}$ solution were performed. As clearly shown in Fig. 4 , a set of ratedependent CV curves of a supercapacitor based on the NiCoP/G hybrid (Fig. 4a) were acquired at various scan rates from 2 to $20 \mathrm{mV} \mathrm{s}^{-1}$ in the potential region of $0.1-0.58 \mathrm{~V}(v s . \mathrm{Hg} / \mathrm{HgO})$. All of the curves exhibit obvious pseudo-capacitance features with 


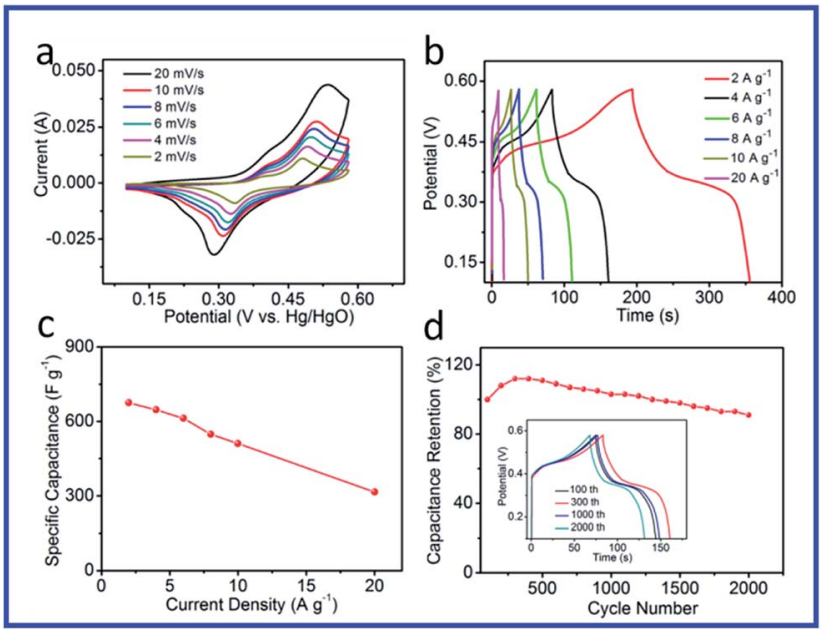

Fig. 4 Electrochemical performances of the NiCoP/G nanostructured electrode: (a) CV results measured at scan rates of 2, 4, 6, 8, 10, $20 \mathrm{mV}$ $\mathrm{s}^{-1}$, (b) galvanostatic charge-discharge curves, (c) specific capacitances at various current densities, (d) evaluation of specific capacitance versus the number of cycles at $4 \mathrm{~A} \mathrm{~g}^{-1}$. The inset is the galvanostatic charge/discharge curve after the 100th, 300th, 1000th and 2000th cycles.

a pair of well-defined redox peaks. Moreover, the peak currents increased with the increase of the scan rate, indicating that the hybrid architectures are beneficial to fast redox reactions. The detailed electrochemical process of the NiCoP/G hybrid on nickel foam substrate at a scanning rate of $2 \mathrm{mV} \mathrm{s}^{-1}$ is illustrated by the CVs in Fig. S8. $\uparrow$ Two pairs of redox peaks can be clearly seen, the first redox couple $\mathrm{A} 1 / \mathrm{C} 1$ is attributed to the conversion between NiCoP and NiCoPOH and the second redox couple $\mathrm{A} 2 / \mathrm{C} 2$ corresponds to the reversible reaction between $\mathrm{NiCoPOH}$ and $\mathrm{NiCoPO},{ }^{48}$ represented by the following reaction:

$$
\begin{gathered}
\mathrm{NiCoP}+\mathrm{OH}^{-} \leftrightarrow \mathrm{NiCoPOH}+\mathrm{e}^{-} \\
\mathrm{NiCoPOH}+\mathrm{OH}^{-} \leftrightarrow \mathrm{NiCoPO}+\mathrm{H}_{2} \mathrm{O}+\mathrm{e}^{-}
\end{gathered}
$$

Fig. $4 \mathrm{~b}$ shows the charge/discharge curves of the NiCoP/G hybrid in the potential range of $0.1-0.58 \mathrm{~V}(v s . \mathrm{Hg} / \mathrm{HgO})$ at various current densities ranging from 2 to $20 \mathrm{~A} \mathrm{~g}^{-1}$. The specific capacitance of the electrode can be calculated from the discharge curve according to the following equation:

$$
C=\frac{I \Delta t}{m \Delta V}
$$

where $C\left(\mathrm{~F} \mathrm{~g}^{-1}\right)$ is the specific capacitance, $I(\mathrm{~A})$ is the charge/ discharge current, $\Delta t(\mathrm{~s})$ is the discharge time, $m(\mathrm{~g})$ is the mass of the active material and $\Delta V(\mathrm{~V})$ is the voltage interval of the discharge. The specific capacitances calculated from the discharge curves were $675,646,612,548,510$, and $315 \mathrm{~F} \mathrm{~g}^{-1}$ at current densities of $2,4,6,8,10$ and $20 \mathrm{~A} \mathrm{~g}^{-1}$, respectively (Fig. 4c). As expected, the $\mathrm{NiCoP} / \mathrm{G}$ electrode displayed better performance compared with the pure NiCoP electrode (Fig. S7b $\dagger$ ). Such superior pseudo-capacitive performance might be attributed to the advantageous structure of the NiCoP NPs assembled on graphene. Small-size particles give rise to high surface area, providing numerous electro-active sites for the redox reaction, while graphene ensures the conductivity of the hybrid material.

The long-term cycling stability of the supercapacitor was tested through a cyclic charge-discharge process at a current density of $4 \mathrm{~A} \mathrm{~g}^{-1}$ (Fig. 4d). Before activation, the NiCoP/G hybrid exhibited pseudo-capacitances of $577 \mathrm{~F} \mathrm{~g}^{-1}$. After activation for 300 cycles, the discharge specific capacitance increased greatly to $646 \mathrm{~F} \mathrm{~g}^{-1}$. After 2000 charge-discharge cycles, the specific capacitances for the $\mathrm{NiCoP} / \mathrm{G}$ electrode decreased to $525 \mathrm{~F} \mathrm{~g}^{-1}$. Hence, the specific capacitance of the $\mathrm{NiCoP} / \mathrm{G}$ hybrid progressively rises to $112 \%$ with the increase of the number of cycles up to the 300th cycle. Moreover, the device still remains at $91 \%$ of the initial capacitance after 2000 chargedischarge cycles, demonstrating an excellent long-term cycling stability. These data highlight the capability of the NiCoP/G hybrid electrode to meet the requirements of practical energy storage devices.

\section{Conclusions}

In summary, a planar hybrid nanostructure of monodisperse NiCoP NPs assembled on graphene has been successfully synthesized. The well-defined NiCoP/G hybrid exhibits excellent lithium storage and supercapacitor properties, which can be attributed to their unique structure. Specifically, the hybrid structure of carbon and nanoparticles effectively improves the structural stability by buffering the volume variation during the cycling process and enhances the conductivity of the electrode material. All these merits would undoubtedly contribute to the electrochemical performance of the electrode materials, leading to high capacity, long cycle life, and superior rate performance for lithium-ion batteries and supercapacitors. This work provides a facile strategy for the synthesis of phosphide particles assembled on graphene for high performance energy storage and conversion.

\section{Acknowledgements}

We acknowledge the support from the National Natural Science Foundation of China (21571166 and 51271173), the National Basic Research Program of China (2012CB922001) and the Fundamental Research Funds for the Central Universities (WK6030000019).

\section{Notes and references}

1 A. J. Bard and M. A. Fox, Acc. Chem. Res., 1995, 28, 141-145. 2 M. W. Kanan and D. G. Nocera, Science, 2008, 321, 10721075.

3 S. Chen, J. W. Zhu, X. D. Wu, Q. F. Han and X. Wang, ACS Nano, 2010, 4, 2822-2830.

4 Z. J. Fan, J. Yan, L. J. Zhi, Q. Zhang, T. Wei, J. Feng, M. L. Zhang, W. Z. Qian and F. Wei, Adv. Mater., 2010, 22, 3723-3728.

5 A. S. Arico, P. Bruce, B. Scrosati, J. M. Tarascon and W. V. Schalkwijk, Nat. Mater., 2005, 4, 366-377. 
6 S. I. Park, Y. Xiong, R. H. Kim, P. Elvikis, M. Meitl, D. H. Kim, J. Wu, J. Yoon, C. J. Yu, Z. Liu, Y. Huang, K. C. Hwang, P. Ferreira, X. Li, K. Choquette and J. A. Rogers, Science, 2009, 325, 977-981.

7 S. Ju, J. Li, J. Liu, P. C. Chen, Y. G. Ha, F. Ishikawa, H. Chang, C. Zhou, A. Facchetti, D. B. Janes and T. J. Marks, Nano Lett., 2007, 8, 997-1004.

8 Y. Y. Mi, W. Liu, K. R. Yang, J. B. Jiang, Q. Fan, Z. Weng, Y. R. Zhong, Z. S. Wu, G. W. Brudvig, V. S. Batista, H. H. Zhou and H. L. Wang, Angew. Chem., Int. Ed., 2016, 55, 14818-14822.

9 N. Choudhary, C. Li, H. S. Chung, J. L. Moore, J. Y. Thomas and Y. Jung, ACS Nano, 2016, 10, 10726-10735.

10 D. H. Youn, S. K. Stauffer, P. H. Xiao, H. M. Park, Y. J. Nam, A. Dolocan, G. Henkelman, A. Heller and C. B. Mullins, ACS Nano, 2016, 10, 10778-10788.

11 M. Acerce, D. Voiry and M. Chhowalla, Nat. Nanotechnol., 2015, 40, 313-318.

12 C. K. Zhang, H. Q. Song, C. F. Liu, Y. G. Liu, C. P. Zhang, X. H. Nan and G. Z. Cao, Adv. Funct. Mater., 2015, 25, 3497-3504.

13 X. Y. Feng, Q. Shen, Y. C. Shi and J. X. Zhang, Electrochim. Acta, 2016, 220, 391-397.

14 C. Zhou, Y. W. Zhang, Y. Y. Li and J. P. Liu, Nano Lett., 2013, 13, 2078-2085.

15 J. W. Hall, N. Membreno, J. Wu, H. Celio, R. A. Jones and K. J. Stevenson, J. Am. Chem. Soc., 2012, 134, 5532-5535.

16 S. Y. Lim, W. Shen and Z. Q. Gao, Chem. Soc. Rev., 2015, 44, 362-381.

17 A. J. Nozik, M. C. Beard, J. M. Luther, M. Law, R. J. Ellingson and J. C. Johnson, Chem. Rev., 2010, 110, 6873-6890.

18 L. F. Cui, Y. Yang, C. M. Hsu and Y. Cui, Nano Lett., 2009, 9, 3370-3374.

19 L. Q. Mai, F. Yang, Y. L. Zhao, X. Xu, L. Xu and Y. Z. Luo, Nat. Commun., 2011, 2, 381-385.

20 H. Chen, L. F. Hu, M. Chen, Y. Yan and L. M. Wu, Adv. Funct. Mater., 2014, 24, 934-942.

21 B. Jang, M. Park, O. B. Chae, S. J. Park, Y. Kim, S. M. Oh, Y. Z. Piao and T. J. Hyeon, J. Am. Chem. Soc., 2012, 134, 15010-15015.

22 R. Bhandavat, L. David and G. J. Singh, J. Phys. Chem. Lett., 2012, 3, 1523-1530.

23 Y. R. Zhu, Z. B. Wu, M. J. Jing, W. X. Song, H. S. Hou, X. M. Yang, Q. Y. Chen and X. B. Ji, Electrochim. Acta, 2014, 149, 144-151.

24 C. Z. Yuan, J. Y. Li, L. R. Hou, J. D. Lin, X. G. Zhang and S. L. Xiong, J. Mater. Chem. A, 2013, 1, 11145-11151.

25 G. J. Hu, C. Xu, Z. H. Sun, S. G. Wang, H. M. Cheng, F. Li and W. C. Ren, Adv. Mater., 2016, 28, 1603-1609.

26 X. F. Wang, B. Liu, Q. F. Wang, W. F. Song, X. J. Hou, D. Chen, Y. B. Cheng and G. Z. Shen, Adv. Mater., 2013, 25, 1479-1486.
27 J. Cabana, L. Monconduit, D. Larcher and M. R. Palacín, Adv. Mater., 2010, 22, E170-E192.

28 C. D. Wang, T. Ding, Y. Sun, X. L. Zhou, Y. Liu and Q. Yang, Nanoscale, 2015, 7, 19241-19249.

29 Y. Lu, X. L. Wang, Y. J. Mai, J. Y. Xiang, H. Zhang, L. Li, C. D. Gu, J. P. Tu and S. X. Mao, J. Phys. Chem. C, 2012, 116, 22217-22225.

30 D. Yang, J. X. Zhu, X. H. Rui, H. T. Tan, R. Cai, H. E. Hoster, D. Y. W. Yu, H. H. Hng and Q. Y. Yan, ACS Appl. Mater. Interfaces, 2013, 5, 1093-1099.

31 D. C. S. Souza, V. Pralong, A. J. Jacobson and L. F. Nazar, Science, 2002, 296, 2012-2015.

32 Y. Lu, J. P. Tu, Q. Q. Xiong, J. Y. Xiang, Y. J. Mai, J. Zhang, Y. Q. Qiao, X. L. Wang, C. D. Gu and S. X. Mao, Adv. Funct. Mater., 2012, 22, 3927-3935.

33 S. L. Liu, C. L. Ma, L. B. Ma and H. Z. Zhang, Chem. Phys. Lett., 2015, 638, 52-55.

34 Z. L. Schaefer, M. L. Gross, M. A. Hickner and R. E. Schaak, Angew. Chem., Int. Ed., 2010, 49, 7045-7048.

35 L. L. Wang, J. W. Liang, Y. C. Zhu, T. Mei, X. Zhang, Q. Yang and Y. T. Qian, Nanoscale, 2013, 5, 3627-3631.

36 J. Chen, J. Z. Wang, A. I. Minett, Y. Liu, C. Lynam, H. K. Liu and G. G. Wallace, Energy Environ. Sci., 2009, 2, 393-396.

37 Z. S. Wu, A. Winter, L. Chen, Y. Sun, A. Turchanin, X. L. Feng and K. Müllen, Adv. Mater., 2012, 24, 5130-5135.

38 B. J. Li, H. Q. Cao, J. Shao, G. Q. Li, M. Z. Qu and G. Yin, Inorg. Chem., 2011, 50, 1628-1632.

39 S. J. Guo and S. H. Sun, J. Am. Chem. Soc., 2012, 134, 24922495.

40 J. J. Yoo, K. Balakrishnan, J. S. Huang, V. Meunier, B. G. Sumpter, A. Srivastava, M. Conway, A. L. M. Reddy, J. Yu, R. Vajtai and P. M. Ajayan, Nano Lett., 2011, 11, 1423-1427.

41 Z. Weng, Y. Su, D. W. Wang, F. Li, J. H. Du and H. M. Cheng, Adv. Energy Mater., 2011, 1, 917-922.

42 Z. Wang, X. Q. Cao, D. N. Liu, S. Hao, G. Du, A. M. Asiri and X. P. Sun, Chem. Commun., 2016, 52, 14438-14441.

43 Y. Z. Su, Q. Z. Xu, G. F. Chen, H. Cheng, N. Li and Z. Q. Liu, Electrochim. Acta, 2015, 174, 1216-1224.

44 Y. J. Bai, H. J. Zhang, L. Liu, H. T. Xu and Y. Wang, Chem.Eur. J., 2016, 22, 1021-1029.

45 A. P. Grosvenor, S. D. Wik, R. G. Cavell and A. Mar, Inorg. Chem., 2005, 44, 8988-8998.

46 Q. Liu, J. Q. Tian, W. Cui, P. Jiang, N. Y. Cheng, A. M. Asiri and X. P. Sun, Angew. Chem., Int. Ed., 2014, 53, 6710-6714.

47 Y. Lu, J. P. Tu, Q. Q. Xiong, H. Zhang, C. D. Gu, X. L. Wang and S. X. Mao, CrystEngComm, 2012, 14, 8633-8641.

48 Y. M. Hu, M. C. Liu, Q. Q. Yang, L. B. Kong and L. Kang, J. Energy Chem., 2017, 26, 49-55. 